\title{
Erratum to: Policy Analytics, Modelling, and Informatics
}

J. Ramon Gil-Garcia, Theresa A. Pardo, and Luis F. Luna-Reyes

\section{Erratum to: \\ J.R. Gil-Garcia et al. (eds.), Policy Analytics, Modelling, and Informatics, Public Administration and Information Technology 25, DOI 10.1007/978-3-319-61762-6}

The volume number for this book was incorrect in the originally published edition as " 24 ". It has been revised in the current version of the book to reflect the actual volume number as " 25 " throughout the book. 